\title{
Not Every Limp in Childhood is Arthritis
}

\section{David Ruiz Picazo ${ }^{1 *}$, Ana Belén Delgado García ${ }^{2}$ and Jorge V Sotoca Fernández ${ }^{3}$}

${ }^{1}$ Department of Orthopedic Surgery, University Hospital of Albacete, Spain

${ }^{2}$ Department of Pediatrics, University Hospital Complex Albacete, Spain

${ }^{3}$ Department of Pediatrics, Malarsjukhuset, Eskilstuna, Sweden

Keywords: Arthritis; Limp; C-reactive protein; Erythrocyte Sedimentation Rate (ESR)

\section{Introduction}

Musculoskeletal pain is a frequent cause of morbidity in children and it usually presents in small child as limp [1]. Its causes are multiple, most often benign, but their identification and differentiation of potentially serious diseases is essential to establish adequate treatment and avoid unnecessary scans [2]. In a large percentage of cases the diagnosis is made with a detailed history, a thorough physical examination and minimum complementary tests [3].

Arthritis is a nonspecific inflammation of one or more joints. It manifests as pain, swelling, local inflammation and reduced joint movement. Its incidence in childhood/adolescence is approximately 403 per 100,000 in US [4]. Limping in children is fairly common in emergency departments. When arthritis settles in the lower limbs the only symptom can be limping or refuse to walk. Then is when we must raise the differential diagnosis with other conditions such as infection, trauma, systemic diseases or tumors [5].

We report the case of a 20-month male which was limping during a week. Through the clinical suspicion, the presence of anodyne lab tests and characteristic images led to the diagnosis. Performed 1 year followup without new events.

\section{Case Report}

We present the case of a 20-months-old male with no relevant family and personal background. He was taken to emergency department when he was limping for a week. No other associated symptomatology. He had no infectious episode recently. The physical examination was normal except for the presence of limitation for full extension and flexion of the left knee without local inflammatory signs. C-Reactive Protein (CRP) and Erythrocyte Sedimentation Rate (ESR) were slightly elevated. The rest of the laboratory tests performed included blood culture was negative. The initial radiography showed no finding (Figure 1). Analgesia was prescribed and after a week the same symptoms persisted. Magnetic Resonance (MR) was performed one month after the onset of symptoms. It presented changes consistent with subacute infectious process in the distal metaphysis of the left femur (Figure 2). Subsequent radiographs showed an osteolytic defect with sclerotic rim (Figure 3). The diagnosis was focal subacute osteomyelitis localized in the left distal femur, called Brodie abscess. The patient was treated with oral amoxicillin-clavulanate and ibuprofen for 4 weeks with excellent clinical and radiological evolution (Figure 4). During the follow-up the patient remained asymptomatic.

\section{Discussion}

Limping is not normal in children, and it deserves a full evaluation. The causes of limping, which are often age-specific and vary widely, include congenital and developmental conditions, infection, inflammation, trauma, systemic illnesses, and tumors [4].
The form of onset (acute or insidious), evolution time (a few hours or days versus weeks or months), duration (continuous or intermittent), intensity, modifying factors (that worsens or improves it), interference with the function (with ambulation), and presence of pain at night, are important data for a first approach to the differential diagnosis [6].

The existence of general symptoms such as fever, weight loss,

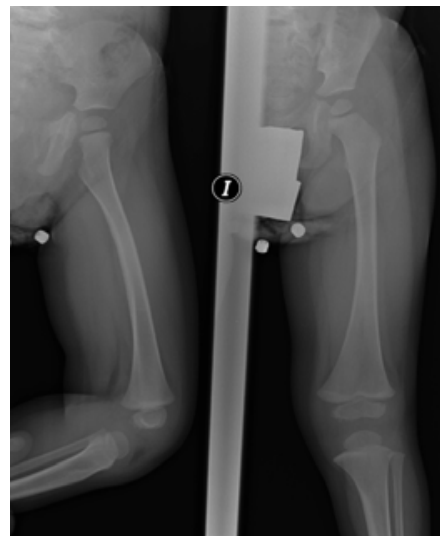

Figure 1: Initial AP and lateral X-ray. No bony alterations are seen on radiographs.

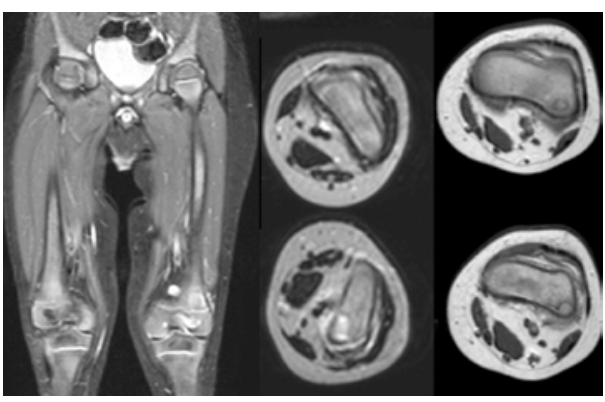

Figure 2: MR. It showed changes consistent with subacute infectious process in the distal metaphysis of the left femur. Observed the ovoid central portion with high signal intensity with a low signal intensity rim on both T1 and T2weighted sequences.

*Corresponding author: David Ruiz Picazo, University Hospital of Albacete street brothers falcó S / N 02006, Albacete, Spain, Tel: +34 666243010, Fax: +34967597290; E-mail: davidcop85@gmail.com

Received February 10, 2014; Accepted February 25, 2014; Published February 27,2014

Citation: Picazo DR, García ABD, Fernández JVS (2014) Not Every Limp in Childhood is Arthritis. J Arthritis 3: 122. doi:10.4172/2167-7921.1000122

Copyright: (c) 2014 Picazo DR, et al. This is an open-access article distributed under the terms of the Creative Commons Attribution License, which permits unrestricted use, distribution, and reproduction in any medium, provided the original author and source are credited. 


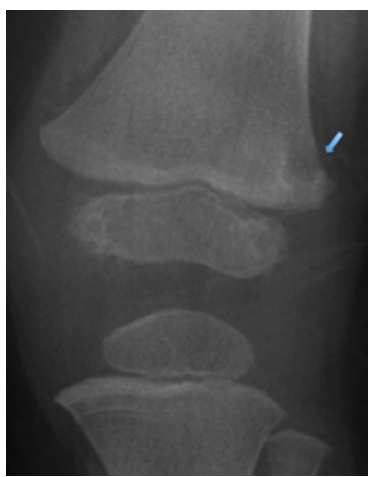

Figure 3: Radiographs performed 2 weeks after the symptoms. Osteolytic defect with sclerotic rim localized on distal metaphysis of left femur (arrow).

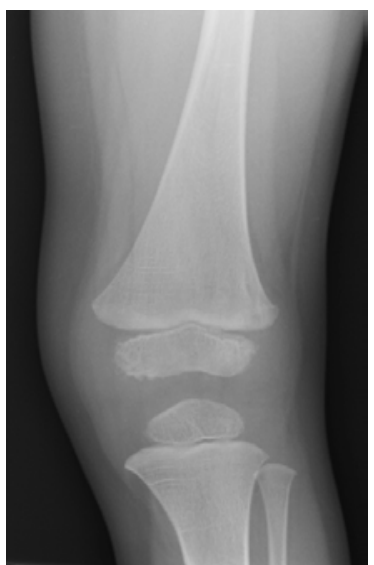

Figure 4: AP X-ray performed one month after starting treatment. Radiological improvement was observed.

fatigue, muscular weakness, anorexia, force to rule out a tumor, infectious or inflammatory disease [7].

The physical examination must be thorough because the osteoarticular pain is a common manifestation of systemic disease [4]. It is important to inspect the skin, make a proper abdominal palpation to rule out visceromegalies and investigate possible lymphadenopathy. Neurological examination and muscle strength are important explorations [3].

The use of complementary tests depends on the presumptive diagnosis we have reached after the history and physical examination. Sometimes a clinical follow up is only necessary [8]. In the patient presented it was planned to treat with ns aids due to no alarm symptom where found. At other times a complete blood count, ESR and CRP to rule out infectious or systemic process is required. If the pain is localized, usually anteroposterior and lateral radiographs of the affected area will be done, also being convenient to do radiographs of the contralateral area, which will help us rule out alleged pathological images and interpret them as normal, common in childhood [2]. A further aspect to take into consideration is timing. Normal radiology in the first days doesn't exclude the pathology [9] as it happened with the case presented. The scintigraphy is helpful in cases of poorly localized pain as often occurs in infants or when osteomyelitis is suspected and no radiological alteration appreciate. This technique is also useful in some tumors of difficult visualization by conventional radiology as osteoid osteoma, prior to other more specific radiological test: Computed Tomography (CT) or MR. In our case, the initial radiographs and laboratory tests were practically normal and the evolution wasn't appropriate so had to be necessary to perform other tests. In the MR will appreciate changes consistent with subacute infectious process localized in the distal metaphysis (ovoid area of high signal in the distal femur with surrounding marrow, muscle, and subcutaneous edema). One osteolytic defect was seen in subsequent radiographs with scleral rim that made us think about a Brodie abscess. More specifically, PET (positron emission tomography) is effective in detecting and monitoring infectious and inflammatory bone diseases. Many investigators have reported the use of FDG-PET (fludeoxyglucose-PET) in the evaluation of osteomielitis and it has been proved in Brodie Abscess [10].

Brodie Abscess represents a form of subacute/chronic osteomielitis with the formation of an intramedullary abscess [10,11]. Involvement of the epiphysis is rare as arthritis (due to the barrier that is the growth plate) [8]. Up to $50 \%$ of cases the isolated microorganism is S. Aureus, followed by Streptococcus, Pseudomonas and Klebsiella $[8,11,12]$. Fungal and mycobacterial infections are rare, mainly seen in immunocompromised patients $[13,14]$.

Organisms typically find their way to the marrow space through a hematogenous route during an episode of transient asymptomatic bacteremia [15]. Thus, Brodie abscess development in the growing child will typically have a predilection for highly vascularized areas of rapid growth, such as long-bone metaphyses, particularly those of the femur and tibia [8]. Brodie abscess is typically unilateral but has been shown in a few case reports to present as bilateral and symmetric lesions.

The differential diagnosis should be established with causes of limping in childhood [2]. The most common cause of limp in childhood is transient synovitis of the hip. Other conditions that should be ruled out include Perthes disease or slipped capital femoral epiphysis [3]. On the other hand, in situations where there is an absence of clinical signs in conjunction with unrevealing laboratory studies, the differential diagnosis can be broad and include other etiologies such as benign lesion as bone cyst or non-ossifying fibroma or malignant injuries as Ewing sarcoma.

The definitive diagnosis is histologic by biopsy of the lesión $[11,12,15]$. The pathological anatomy is characterized by granulation tissue with leukocyte infiltrates, histiocytes and capillary revascularization. Also it can be found areas of necrotic trabecular bone with fibrin in the medullary cavity [16]. Nevertheless, a consistent clinical presentation with typical radiology and successful course could be enough and biopsy avoided.

Treatment of Brodie abscess is based on two pillars, medical and surgical. The patients usually respond well after surgical resection of the cavity and the introduction of antibiotic treatment. Sometimes, bone grafting is needed especially in those lesions larger than $3 \mathrm{~cm}$ [11]. It has been seen that most children with less aggressive presentations and no evidence of subperiosteal abscess can be treated exclusively with medical therapy and immobilization $[8,11,14-16]$. The child here presented had approximately one month without deterioration or new manifestations until RM was performed. With the typical radiological findings the diagnose was establish. Neither surgical treatment nor biopsy was accomplished with clinical and radiological resolution of the disease. 


\section{Conclusion}

Musculoskeletal pain is a common cause of morbidity in children and may be presented in childhood as a limp. Frequently it is trauma or a self-limiting inflammatory process the cause. However, it is forgotten other causes of musculoskeletal pain as tumors or infection. Osteomyelitis is the most common childhood osteoarticular infection. Brodie's abscess is a type of subacute localized hematogenous osteomyelitis. We must think about it when a child has a selective and localized pain in a limb without history of trauma and unresponsive to analgesic treatment. Results with medical only or medical-surgical treatment are typically excellent.

\section{References}

1. Jacobs JC (1982) The differential diagnosis of arthritis in childhood. En Pediatric Rheumatology for the Practitioner. $\left(2^{\text {nd }}\right.$ edn) New York, SpringerVerlag 25-230.

2. Garcia-Consuegra Molina J, Merino Munoz R (2000) Sindromes Dolorosos Musculo-esqueleticos en la infancia. Medicine 8: 1433-1439.

3. Sawyer JR1, Kapoor M (2009) The limping child: a systematic approach to diagnosis. Am Fam Physician 79: 215-224.

4. Sacks JJ1, Helmick CG, Luo YH, llowite NT, Bowyer S (2007) Prevalence of and annual ambulatory health care visits for pediatric arthritis and other rheumatologic conditions in the United States in 2001-2004. Arthritis Rheum 57: $1439-1445$
5. Gunner KB1, Scott AC (2001) Evaluation of a child with a limp. J Pediatr Health Care 15: 38-40.

6. Turrion Nieves Al, Martin Holguera R, Perez Gomez A, Sanchez Atrio Al (2009) Protocolo diagnostico de una artritis intermitente. Medicine 10: 1969-197.

7. Daz- Delgado, Peas R (2006) Protocol Diagna-diagnosis and Pharmacologic utico of arthritis in adolescence. Medicine 9: 3982-3985.

8. Abdulhadi MA1, White AM, Pollock AN (2012) Brodie abscess. Pediatr Emerg Care 28: 1249-1251.

9. Harris NH, Kirkaldy-Willis WH (1965) Primary Subacute Pyogenic Osteomyelitis J Bone Joint Surg Br 47: 526-532.

10. Strobel K1, Hany TF, Exner GU (2006) PET/CT of a brodie abscess. Clin Nucl Med 31: 210.

11. Alter SA1, Sprinkle RW (1995) Brodie's abscess: a case report. J Foot Ankle Surg 34: 208-214.

12. Ross ER, Cole WG (1985) Treatment of subacute osteomyelitis in childhood. J Bone Joint Surg Br 67: 443-448.

13. McCarthy JJ, Dormans JP, Kozin SH, Pizzutillo PD (2004) Musculoskeletal infections in children. J Bone Joint Surg 86: 850-863.

14. Rasool MN (2001) Primary subacute haematogenous osteomyelitis in children. J Bone Joint Surg Br 83: 93-98.

15. Chambler AF1, Chapman-Sheath PJ, Pearse MF, Hollingdale J (1997) Symmetrical Brodie's abscess. Postgrad Med J 73: 660-661.

16. Moragues Sbert G, Soleto Roncero MJ, del Rio Mangada A, Toribio Pons JA (2006) Absceso de Brodie. Acta Pediatr Esp 64: 443-445. 